\title{
Coaching im Mittelstand
}

\section{Cornelia Seewald · Astrid Schreyögg}

Der Schwerpunkt des vorliegenden Heftes widmet sich dem Thema Coaching im Mittelstand. Die zunehmende Professionalisierung und Akademisierung von Coaching zeigt sich einerseits in einer wachsenden Zahl wissenschaftlicher Arbeiten und konzeptioneller Ansätze und entsprechender verbandsübergreifender Standards und andererseits in einer transparenter werdenden Segmentierung der Anwendungsfelder. Während konzeptionelle Coaching-Programme in globalen Konzernen längst zum HR Portfolio gehören und der individualisierte Markt der Nachfrage nach Coaching Blüten treibt, gibt es bisher eher wenige mittelstandsspezifische Ansätze und Programme zum Thema. Letzteres liegt in der Zielgeraden des Fachausschusses Mittelstand des DBVC, aus dem zu diesem Schwerpunktheft fünf Beiträge vorliegen.

Gudrun Happich und Marcus Classen beschreiben die Heterogenität des Mittelstandes unter dem Gesichtspunkt der Zielgruppen- und Bedarfsspezifikation und in Abgrenzung zur Situation der Konzerne. Sie reflektieren das Profil eines Coachs, der im Mittelstand agiert, und illustrieren dies durch kurze Praxisbeispiele. Birgitta Fildhaut berichtet aus ihrer Erfahrung mit Doppelspitzen-Coaching in einem Familienunternehmen eine Konstellation, die neben anderen als typisch für den Mittelstand gelten darf. In ihrem Beitrag wird deutlich, wie sehr historisch tradiertes Beziehungsgeschehen in den unternehmerischen Alltag hineinwirken kann. Und es wird deutlich, auf welche Weise Coaching zur Entlastung der Akteure und deren Neuorientierung beitragen kann. Eine ganz andere Perspektive erläutert Wolfgang Filbert. Er beantwortet nicht nur die Frage: Rechnet sich Coaching im Mittelstand?, sondern auch: Welchen Zusammenhang gibt es zwischen gesundheitsorientiertem Führungs-Coaching und betriebswirtschaftlichem Unternehmenserfolg? Er tritt den Beweis an und erläutert ihn an einem konkreten Beispiel. Welche Unterstützung brauchen Start ups? Cornelia Seewald beschreibt die Interventionen eines rollenflexiblen Coachs einer kleinen Werbeagentur während der kritischen Phase im dritten Jahr nach der Gründung. Wo institutionelle Fördermaßnahmen enden, aber die unternehmerische Solidität noch nicht erreicht ist, kann Komplementärberatung - auch

\footnotetext{
Online publiziert: 12.07 .2013

(C) Springer Fachmedien Wiesbaden 2013

Dr. A. Schreyögg $(\bowtie)$

Breisgauer Str. 29, 14129 Berlin, Deutschland

E-Mail: info@schreyoegg.de

Dr. C. Seewald

Sternstr. 32, 40479 Düsseldorf, Deutschland
} 
durch einen Berater allein - das Schlimmste abwenden. Im fünften Schwerpunkt-Beitrag dann erläutert Gudrun Happich, wie der Mittelstand Leistungsträger binden kann: Monetäre Absicherung, explizite Wertschätzung und verbindliche Entwicklungschancen verhelfen dem Mittelstand auf dem Arbeitsmarkt zu Wettbewerbsvorteilen. Coaching als individuelle Maßnahme, aber strategisch eingesetzt, kann dabei angemessen Unterstützung leisten.

Im Sinne einer guten Tradition von OSC präsentieren wir neben Aufsätzen zu einem Schwerpunktthema noch einige zusätzliche Beiträge. Im ersten beschäftigt uns Monika Klinkhammer mit Coaching von Wissenschaftler/innen. Sie gibt uns aus einer Hochschule in Berlin einen Einblick in die enorm vielfältigen Belastungen und Rollenanforderungen dieses Feldes. Deshalb steigt ihrer Meinung nach auch hier der Coaching-Bedarf in unterschiedlichen Karrierestadien laufend an. Der nachfolgende Beitrag ist einem methodischen Thema gewidmet. Theresa Schomaker, Charlotte von Bernsdorf und Jens Nachtwei haben zur Stressreduktion von Führungskräften in Unternehmen ein spezifisches Training mit Outdoor-Elementen konzipiert. Dieses soll die Teilnehmer/innen in ihren Coping-Strategien gegenüber beruflichem Stress nachhaltig stärken. Daran anschließend erschließt uns Thies Carstens die methodischen Möglichkeiten von Tablet-Computern fürs Coaching. Er betont, dass PCs den Face-to-face-Kontakt zwar nicht ersetzen können, dass sie aber für die Vor- und Nachbereitung sowie für die Begleitung der jeweiligen Gespräche eine Vielzahl an zusätzlichen methodischen Möglichkeiten enthalten. Im Diskurs konfrontiert uns Ulrike Stöwer aus einer linguistischen Perspektive mit „Beratersprache im historischen Kontext". Die Autorin zeigt uns am Beispiel der Verben anbieten und einladen, welche Bedeutungen die beiden Wörter eigentlich beinhalten. Dies kann dazu dienen, den bewusst mit Sprache umgehenden Berater auf neue Spuren des Sprachverständnisses zu bringen. 